\title{
Enfermedad de Hansen: Revisión a propósito de un caso
}

Marcela Concha R., M. Laura Cossio T., Iván Salazar S., Félix Fich S., Carlos Pérez C. y Sergio González B.

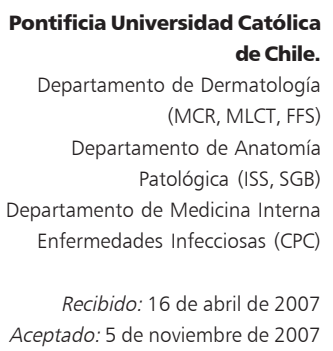

Pontificia Universidad Católica

de Chile.

Departamento de Dermatología (MCR, MLCT, FFS)

Departamento de Anatomía

Patológica (ISS, SGB)

Departamento de Medicina Interna

Enfermedades Infecciosas (CPC)

Recibido: 16 de abril de 2007 Aceptado: 5 de noviembre de 2007

Correspondencia a: Marcela Concha Rogazy marcelaconchar@gmail.com

\section{Hansen`s disease: Case report and review of literature}

Leprosy is a granulomatous disease affecting the skin and peripheral nerves caused by Mycobacterium leprae. The range of clinical forms varying from tuberculoid to lepromatous leprosy results from variations in the cellular immune response to the mycobacterium. Despite available combined drug-therapy, it continues to be a significant public health problem, carrying a strong stigma. Although recently there has been no native cases in Chile, a few imported cases have been diagnosed. We present a 56-year-old man who had lived in Paraguay for 8 years, and presented with leprosy 6 years after returning to Chile. The biology of leprosy, clinical features of the disease, current diagnostic criteria and approaches to treatment are discussed.

Key words: Hansen's disease; leprosy; granulomatous disease.

Palabras clave: Enfermedad de Hansen; lepra; enfermedad granulomatosa.

\section{Introducción}

$\mathrm{L}$ a enfermedad de Hansen, más conocida como lepra, es una infección granulomatosa crónica, producida por el bacilo Mycobacterium leprae, que compromete, primariamente, la piel y los nervios periféricos. Los primeros casos de lepra se describieron en el año 600 AC en la India, pero sólo se conoce la causa desde 1873, cuando el noruego Gerhard Hansen identificó al $M$. leprae como agente etiológico ${ }^{1}$.

Aunque rara vez es mortal, las complicaciones secundarias a la neuropatía pueden ser deformantes e incapacitantes. La terapia combinada utilizada por la OMS desde 1983, ha demostrado que la infección puede ser tratada en forma efectiva antes de llegar a la discapacidad. Sin embargo, la lepra sigue siendo una enfermedad estigmatizante, constituyendo un problema de salud pública en países tropicales y subtropicales de América, Asia y África ${ }^{1,2}$.

\section{Caso Clínico}

Hombre de 56 años de edad con antecedentes de un síndrome depresivo mayor, en tratamiento con litio y alprazolam, que consultó por lesiones cutáneas asintomáticas en el antebrazo derecho, de aproximadamente tres años de evolución, asociadas a otras lesiones similares en el tronco y las extremidades inferiores, de tres meses de evolución. El paciente refería hipoestesia leve en algunas placas, sin otros síntomas asociados. Como antecedente, el paciente había resi- dido durante ocho años en Paraguay y había regresado a Chile hacía seis años al momento de consultar.

Al examen físico, el paciente se encontraba en buenas condiciones generales y presentaba placas y nódulos eritematosos e infiltrados en las zonas malares, la frente, nariz, hélix, antebrazos, el tórax anterior y posterior y los muslos, de distribución simétrica (Figuras 1 y 2). Además, en el antebrazo derecho, muslo derecho y la espalda se observaban placas anulares, de bordes solevantados y tendencia a la curación central, con leve hipoestesia en el centro (Figuras 3-5). No se objetivó aumento de tamaño de los nervios periféricos ni déficit motor o sensitivo secundarios al daño de troncos nerviosos.

Con el diagnóstico clínico de enfermedad granulomatosa versus linfoma cutáneo, se realizó una biopsia de piel, que mostró una inflamación granulomatosa en la dermis reticular. Los granulomas estaban constituidos por macrófagos, sin caseificación y con infiltrado linfocitario, perifocal. Algunos macrófagos con citoplasma vacuolado. Se distribuían, principalmente, alrededor de los vasos sanguíneos y también de filetes nerviosos, con extensión de linfocitos al endoneuro. La tinción de Kinyoun mostró abundantes bacilos intra y extracelulares, compatibles con M. leprae.

Tanto por las características clínicas como histopatológicas, se catalogó como una lepra borderline lepromatosa, iniciándose el tratamiento gratuito otorgado por el MINSAL (dapsona $100 \mathrm{mg} / \mathrm{d}$, rifampicina $600 \mathrm{mg} / \mathrm{d}$ y clofazimina $300 \mathrm{mg} / \mathrm{mes}$ ), con buena respuesta clínica a los seis meses de tratamiento. Se controlaron sus contactos más directos, sin hallazgos al 

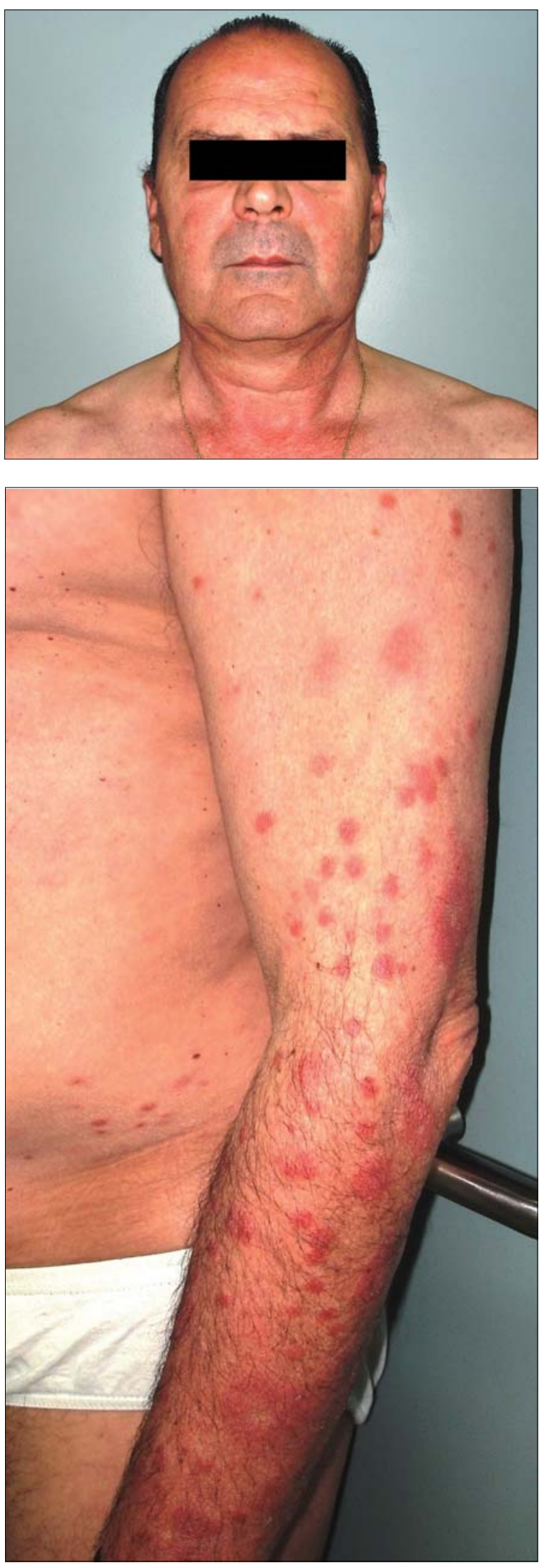

Figura 2. Placas y nódulos eritematosos en el brazo izquierdo.

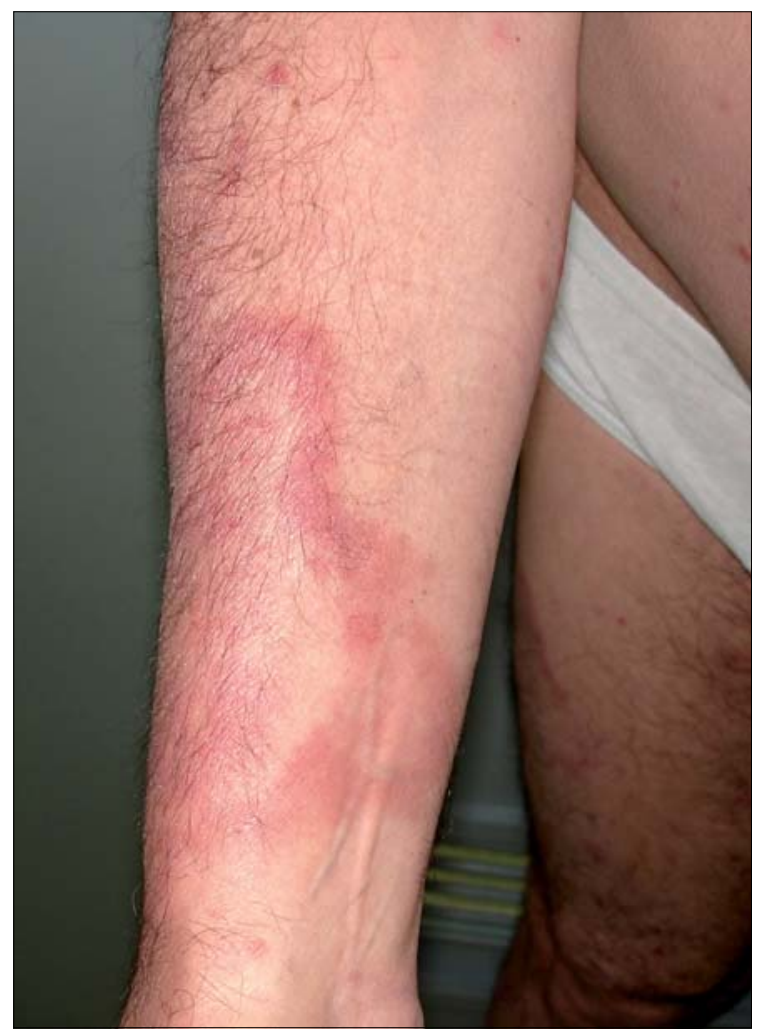

Figura 3. Placas anulares en el antebrazo derecho.

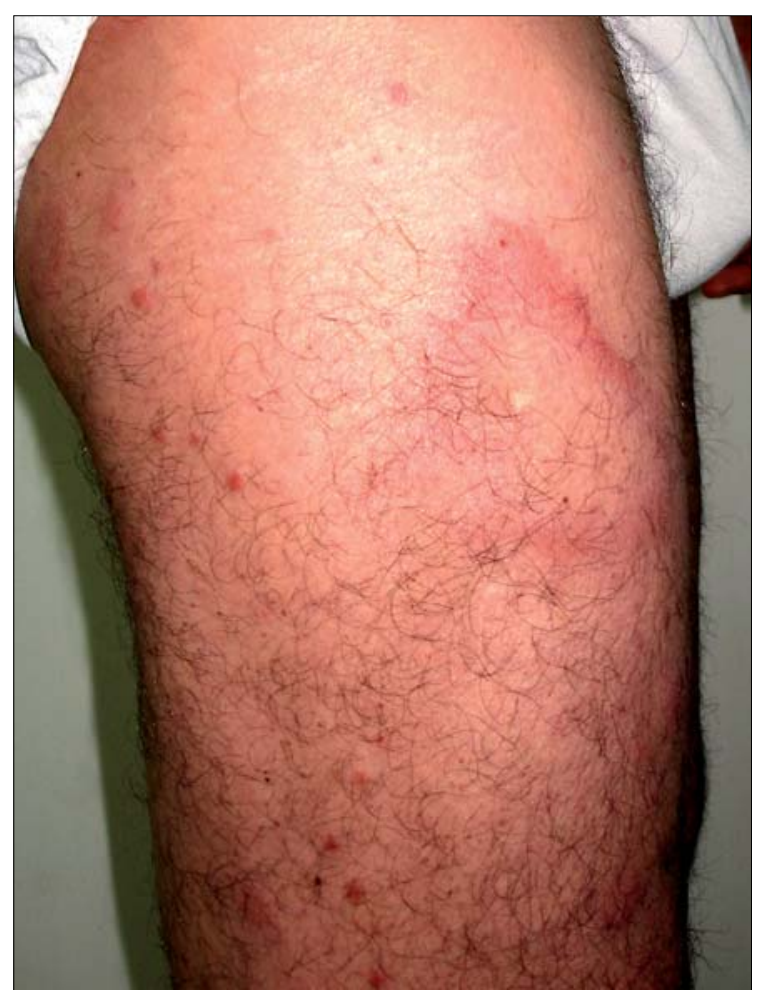

Figura 4. Placas anulares en el muslo derecho. 
Figura 5. Placas anulares en la espalda.

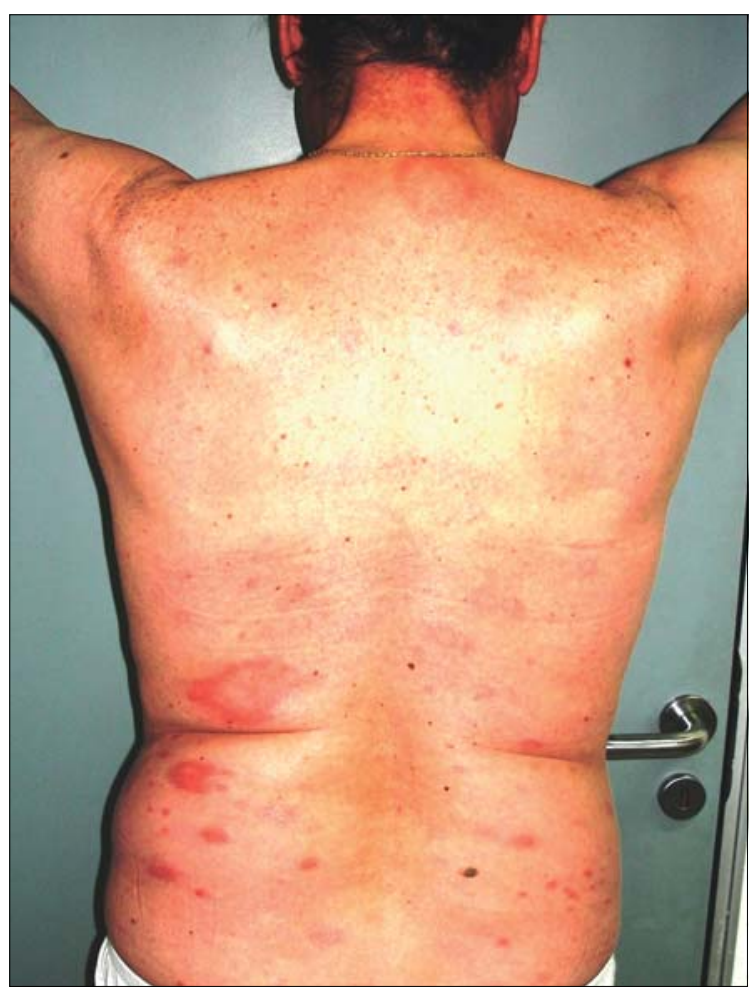

Patogenia. Mycobacterium leprae es un bacilo ácido-alcohol resistente, intracelular obligado. Hasta hoy no ha sido posible cultivar el bacilo in vitro, por lo que el tiempo de replicación se ha estimado, a partir de la inoculación de bacilos viables en patas de ratones, en 12,5 días en promedio, en concordancia con su prolongado período de incubación, que es de aproximadamente tres a cinco años ${ }^{6}$. La temperatura óptima para su reproducción está entre 27 y $33^{\circ} \mathrm{C}$, lo que explicaría su predilección por las áreas más frías del cuerpo como son la piel, los nervios periféricos y la mucosa de la vía aérea superior ${ }^{7}$. En la piel se encuentra preferentemente en los macrófagos, donde, en pacientes sin una respuesta inmune celular, evita ser digerido por la célula, multiplicándose hasta formar grandes masas de bacilos, que finalmente producen rotura de la membrana celular. En el tejido neural se ubica en las células de Schwann, donde puede permanecer por largo tiempo sin estimular una respuesta inmune (sitio de protección inmunológica) ${ }^{6}$.

El mecanismo exacto de transmisión de $M$. leprae es desconocido. Sin embargo, se cree que la diseminación ocurriría por vía respiratoria, de manera similar a la tuberculosis, ya que la secreción nasal de los enfermos multibacilares no tratados contiene gran cantidad de bacilos (medida por técnica de RPC). Por otra parte, se ha observado que el bacilo se mantendría viable hasta 24 horas fuera del organismo, en las gotitas de la secreción nasal, lo que apoya esta hipótesis ${ }^{6,7}$. Sólo existen algunos casos reportados de transmisión en piel dañada (sitios de punción, otras heridas), y no hay evidencia de transmisión por contacto sexual ${ }^{6}$.

La enfermedad de Hansen es un ejemplo clásico de una infección cuyas manifestaciones corresponden a la respuesta inmune del huésped, expresada por un amplio espectro de características clínicas, histológicas e inmunológicas. De hecho, se estima que sólo 3 a 5\% de la población expuesta a $M$. leprae es susceptible de desarrollar la enfermedad, lo que no dependería de la virulencia del bacilo sino de las características inmunológicas del huésped ${ }^{6}$. Dentro de los factores de riesgo conocidos, se encuentra la presencia de un contacto cercano (domiciliario), el tipo de lepra que tenga ese contacto (es mayor el riesgo en la lepra multibacilar), la edad del sujeto (a mayor edad, mayor el riesgo) y las condiciones asociadas a la pobreza como hacinamiento y bajo nivel educacional, entre otras ${ }^{8,9}$. Esto último se relaciona con el comportamiento epidemiológico de la infección, descrito anteriormente.

No se ha demostrado que los pacientes con SIDA tengan una mayor tasa de infección por M. leprae, ni tampoco un comportamiento clínico más grave, a diferencia de lo que ocurre con la tuberculosis ${ }^{10}$. Esto confirma que el mecanismo inmunológico de respuesta 
a M. leprae sería diferente al de M. tuberculosis, y estaría preservado en los pacientes con infección por VIH, aún en etapas avanzadas de inmunosupresión. Sin embargo, la vacunación con BCG ha demostrado tener un efecto protector relativo contra la lepra, que varía entre 26 y 60\%, en los diferentes estudios ${ }^{11}$. Por otra parte, se han descrito casos de lepra en pacientes en tratamiento con antagonistas de FNT- $\alpha$ como infliximab ${ }^{12}$.

La herencia también jugaría un rol en la enfermedad de Hansen. Aunque no se ha identificado una asociación clara entre HLA y susceptibilidad a desarrollar lepra, sí existe relación entre algunos HLA específicos y las formas de presentación de la enfermedad ${ }^{6}$.

Cuadro Clínico. Como se dijo anteriormente, la expresión clínica en la enfermedad de Hansen es un reflejo de la respuesta inmune del huésped y comprende un amplio espectro de manifestaciones. Además de las lesiones cutáneas, el bacilo produce desmielinización segmentaria de los axones de las terminaciones nerviosas de la dermis, y de los troncos nerviosos localizados en sitios periféricos, principalmente los nervios tibial posterior, cubital, mediano, ciático poplíteo externo, facial, auricular mayor y radial ${ }^{2,6}$. Este daño neural es secundario a la inflamación granulomatosa desencadenada por $M$. leprae, y se traduce en disminución de la sensibilidad y sudoración en las lesiones (nervios dérmicos) y déficit motor, sensitivo, autonómico o mixto (troncos nerviosos), pudiendo preceder al compromiso cutáneo en algunos $\operatorname{casos}^{13}$.

La detección de $M$. leprae en la secreción nasal de sujetos asintomáticos, por técnica de RPC, podría ser un indicador de una infección subclínica; sin embargo, aún no se ha demostrado que estos sujetos tengan un mayor riesgo de desarrollar la enfermedad que el resto de la población expuesta al bacilo ${ }^{14}$. Por otra parte, de existir infección subclínica, aún no se conoce el sitio del foco primario ${ }^{6}$.

Actualmente, se utilizan dos clasificaciones (Tabla 1); la de Ridley-Jopling, que utiliza características clínicas e histopatológicas, se divide en seis categorías que se correlacionan con la actividad de la respuesta inmune del huésped. La clasificación de la OMS divide a los pacientes en dos categorías, considerando el número de lesiones cutáneas. Esta última tiene ventajas desde el punto de vista operacional ya que permite definir el tratamiento ${ }^{2}$.

Lepra indeterminada (I). Corresponde a la etapa más temprana, donde las características clínicas e histopatológicas impiden clasificarla. Las lesiones consisten en una o dos máculas hipopigmentadas, con sensibilidad normal o leve hipoestesia, y puede resolverse en forma espontánea o progresar a las etapas siguientes. En la histopatología no hay evidencia de granulomas y el infiltrado inflamatorio es inespecífi$\mathrm{CO}^{1,2,6,7}$.

Lepra tuberculoide (TT). Refleja una buena respuesta inmune del hospedero, caracterizada por la formación de granulomas epiteloideos rodeando los elementos neurovasculares, con un denso infiltrado linfocítico que se extiende hasta la dermis. Clínicamente, se presenta como máculas hipopigmentadas o placas eritematosas, de bordes solevantados y tendencia a la curación central. En esta etapa, el daño de las terminaciones nerviosas de la dermis es precoz, por lo que en las lesiones suele haber marcada disminución de la sensibilidad, además de daño de uno o dos troncos nerviosos cercanos, que pueden estar aumentados de tamaño y asociarse al compromiso sensitivo o motor de la zona inervada ${ }^{1,2,6,7}$.

Lepra lepromatosa (LL). Se encuentra al otro extremo del espectro clínico ya que refleja una respuesta inmune muy débil, encontrándose abundantes bacilos en la piel y la mucosa nasal. Comienza como máculas simétricas, ligeramente eritematosas, mal delimitadas, que progresan a una infiltración generalizada de toda la piel, mayor en las zonas más frías, donde puede haber nódulos, dando en la cara el aspecto de "fascie leonina". Además, hay pérdida de cejas y pestañas, pero rara vez produce alopecia ya que el cuero cabelludo es un sitio de mayor temperatura. Se produce engrosamiento e ictiosis en las piernas y, en ocasiones, la piel afectada puede ulcerarse. Al no haber formación de granulomas, el compromiso neural suele ser tardío pero extenso, y la función motora, en general, está conservada $a^{1,2,6,7}$.

Lepra borderline. Las formas borderline conforman un espectro intermedio entre la tuberculoide, con una buena respuesta inmune del hospedero, y la lepromatosa, con una respuesta casi nula. La borderline tuberculoide (BT) es similar a la tuberculoide, pero las lesiones son más numerosas. La forma borderline lepromatosa se diferencia de la lepromatosa en que las lesiones son más definidas, asimétricas, y con áreas de piel sana entre ellas. La media borderline (BB) es una

Tabla 1. Clasificaciones de Ridley-Jopling y OMS
Clasificación de Ridley-Jopling

Indeterminada (I)

Tuberculoide (TT)

Borderline tuberculoide (BT)

Media borderline (BB)

Borderline lepromatosa (BL)

Lepromatosa (LL)

\section{Clasificación OMS}

Paucibacilar (PB)

1 a 5 lesiones cutáneas

Multibacilar (MB)

$>5$ lesiones cutáneas 
forma inestable, que puede variar a BT o BL, y se caracteriza por la presencia de placas de borde elevado en declive, con islas de piel aparentemente sana, de bordes bien definidos ${ }^{1,2,6,7}$.

Compromiso ocular. La ceguera se describe en $3,5 \%$ de los pacientes con lepra y se produce por daño del $\mathrm{V}^{\mathrm{o}}$ y VII ${ }^{\mathrm{o}}$ nervios craneanos e invasión de la piel y del ojo mismo, que resultan en lagoftalmia (imposibilidad de cerrar el ojo), úlceras corneales, iridociclitis aguda o crónica, cataratas secundarias y opacidad corneal $^{2}$.

Reacción reversa o tipo 1 . Corresponde a una reacción de hipersensibilidad tipo IV, que ocurre hasta en $30 \%$ de los pacientes con formas borderline. Se caracteriza por una inflamación aguda de las lesiones cutáneas, que se hacen más edematosas y eventualmente se ulceran, y por una neuritis aguda, que puede llevar a déficit sensitivo o motor persistente si no se trata. Aunque este tipo de reacción puede observarse en cualquier momento, es más frecuente al iniciarse la terapia $^{1,2,6,7}$.

Reacción eritema nodoso leproso o tipo 2. Afecta al $20 \%$ de las formas lepromatosas y al $10 \%$ de las borderline lepromatosa y es más frecuente mientras mayor sea la infiltración cutánea y el índice bacteriano. Se produce por depósito de complejos inmunes y se asocia a concentraciones elevadas de FNT- $\alpha$. Clínicamente se caracteriza por fiebre y pápulas o nódulos eritematosos, dolorosos, que pueden ser profundos, causando paniculitis y ulceraciones. También puede producir uveítis, neuritis, artritis, dactilitis y orquitis ${ }^{1,2,6,7}$.

Fenómeno de Lucio. Es poco frecuente, se observa en la forma lepromatosa en pacientes de México y Costa Rica, siendo raro en otras regiones. Corresponde a una vasculitis de la dermis, que se manifiesta por placas dolorosas, purpúricas, que tienden a ampollarse y ulcerarse, curando con cicatriz atrófica. En algunos casos se asocia a gran compromiso sistémico, pudiendo ser fatal. Se presenta en pacientes no tratados y su patogenia exacta se desconoce ${ }^{15}$.

Diagnóstico. Se basa en su expresión clínica, con presencia de lesiones cutáneas características, asociadas a pérdida de la sensibilidad o aumento de volumen

Tabla 2. Esquemas terapéuticos de la OMS y Estados Unidos de América

$\begin{array}{llrllr}\text { Lepra } & & \text { Dapsona } & \text { Rifampicina } & \text { Clofazimina } & \text { Duración } \\ \text { Paucibacilar (PB) } & \text { OMS } & 100 \mathrm{mg} / \mathrm{d} & 600 \mathrm{mg} / \mathrm{mes} & & 6 \text { meses } \\ & \text { E.UA. } & 100 \mathrm{mg} / \mathrm{d} & 600 \mathrm{mg} / \mathrm{d} & & 12 \text { meses } \\ \text { Multibacilar (MB) } & \text { OMS } & 100 \mathrm{mg} / \mathrm{d} & 600 \mathrm{mg} / \mathrm{mes} & 50 \mathrm{mg} / \mathrm{d}+ & 12 \text { meses } \\ & & & & 300 \mathrm{mg} / \mathrm{mes} & \\ & \text { E.U.A. } & 100 \mathrm{mg} / \mathrm{d} & 600 \mathrm{mg} / \mathrm{d} & 50 \mathrm{mg} / \mathrm{d} & 24 \text { meses }\end{array}$

de los nervios periféricos en los sitios de elección. La presencia de bacilos ácido-alcohol resistentes en una biopsia y/o frotis de piel también confirma el diagnóstico $^{16}$.

Ni la serología ni la RPC tienen un rol diagnóstico hasta el momento. Los anticuerpos contra PGL-1 (phenolic glycolipid-1), específicos para M. leprae, están presentes en $90 \%$ de los pacientes con lepra lepromatosa no tratada, pero sólo en $50 \%$ de los sujetos con formas paucibacilares y en $5 \%$ de los controles sanos $^{16,17}$. La RPC en tejido tiene una sensibilidad de 95 a $99 \%$ en formas multibacilares y de 50 a $60 \%$ en las paucibacilares, por lo que no se utiliza de rutina en la práctica clínica ${ }^{16,18}$.

Tratamiento. La antibioterapia combinada fue introducida en 1982, después de que se reportara 30\% de resistencia a dapsona. Aunque se han estudiado otros antibacterianos, el tratamiento estándar recomendado por la OMS incluye dapsona, rifampicina y clofazimina ${ }^{16}$.

La dapsona (diamino-difenil sulfona) fue la primera en utilizarse para el tratamiento de la lepra. Su mecanismo de acción sería similar al de otras sulfas, inhibiendo la síntesis de ácido fólico ${ }^{16}$. Aunque se considera un fármaco seguro, puede asociarse a anemia hemolítica grave en pacientes con déficit de glucosa-6-fosfato deshidrogenasa (G-6PD), meta-hemoglobinemia y al síndrome de hipersensibilidad por dapsona, que ocurre en forma idiosincrásica y se caracteriza por fiebre, exantema máculo-papular, adenopatías y hepatitis ${ }^{19}$.

La rifampicina es un bactericida potente, que actúa inhibiendo la ARN polimerasa al unirse a su subunidad- $\alpha$.

La clofazimina es un bactericida débil, cuyo mecanismo de acción se desconoce, pero se cree que tendría algún efecto anti-inflamatorio ya que disminuye la incidencia de reacción tipo eritema nodoso leproso ${ }^{16}$.

El esquema de la OMS difiere del esquema utilizado en E.U.A. en que rifampicina se indica en dosis de una vez al mes, y no diariamente, y también en la duración del tratamiento (Tabla 2). El uso diario de rifampicina en lepra multibacilar se asociaría a una caída, significativamente mayor, en el índice bacteriano que el tratamiento con dosis mensuales ${ }^{20}$. Sin embargo, no existen ensayos clínicos randomizados que demuestren mejores resultados clínicos, y por el elevado costo que tendría implementar la rifampicina en dosis diaria, la OMS sigue recomendando como tratamiento estándar la dosis mensual. En Chile, el MINSAL distribuye gratuitamente la terapia específica.

Estudio y manejo de los contactos. La OMS recomienda realizar examen físico de la piel y los nervios periféricos a todos los contactos domiciliarios al momento del diagnóstico y luego, una vez al año, por 5 años, en casos de lepra multibacilar. 
Quimioprofilaxis de contactos. La mayoría de los estudios realizados con dapsona muestran cerca de $60 \%$ de protección, efecto que disminuiría en el seguimiento a largo plazo. Algunos estudios realizados con rifampicina han mostrado un efecto protector leve, pero falta más evidencia al respecto, por lo que se sugiere sólo tratar el paciente cuando presenta el cuadro clínico ${ }^{21}$.

\section{Resumen}

La lepra es una enfermedad granulomatosa causada por Mycobacterium leprae, que afecta la piel y los nervios. Su espectro clínico comprende desde lepra tuberculoidea hasta la lepromatosa, siendo el resultado de las variaciones de la respuesta inmune celular a la micobacteria. A pesar de la terapia combinada, la enfermedad sigue siendo un problema de salud pública significativo y se asocia a una fuerte estigmatización. Aunque no ha habido casos autóctonos en Chile, algunos casos contraídos en el extranjero han sido diagnosticados. Se presenta un hombre de 56 años, que vivió en Paraguay durante ocho años y debutó con lepra después de seis años viviendo en Chile. Se discute la biología de la lepra, las características clínicas de la enfermedad, los criterios diagnósticos actuales y el enfoque terapéutico.

\section{Referencias}

1.- Ramos-e-Silva M, Ribeiro de Castro M C. Infecciones por micobacterias. En: Dermatología, Bologna J L, Jorizzo J L, Rapini R P, editores. Primera Edición. Madrid, Elsevier España; 2004: 1145-64.

2.- Walter S L, Lockwood D N. Leprosy. Clin Dermatol 2007; 25: 165-72.

3.- Fine P E. Leprosy: what is being "eliminated"? Bull World Health Org 2007; 85: 2.

4.- Global leprosy situation, 2006. Wkly Epidemiol Rec 2006; 81: 309-16.

5.- Zúñiga M. Lepra (Enfermedad de Hansen). En http://epi.minsal.cl/epi/html/enfer/ lepra.htm (Accedido 22 de marzo de 2007).

6.- Yoder L D, Guerra I E. Hansen’s Disease. A guide to management in the United States. Hansen's Disease Foundation 2001.

7.- Jacobson R, Krahenbuhl J, Yoder L. Overview of leprosy. UpToDate 15.1.

8.- Bakker M I, Hatta M, Kwenang A, Van Mosseveld P, Faber W R, Klatser P R, et al. Risk factors for developing leprosy: a population-based cohort study in Indonesia. Lepr Rev 2006; 77: 48-61.

9.- Kerr-Pontes L R, Barreto M L, Evangelista C M, Rodrigues L C, Heukelbach J, Feldmeier H. Socioeconomic, environmental, and behavioural risk factors for leprosy in North-east Brazil: results of a case-control study. Int J Epidemiol 2006; 35: 994-1000.

10.- Ustianowski A P, Lawn S D, Lockwood D N. Interactions between HIV infection and leprosy: a paradox. Lancet Infect Dis 2006; 6: $350-60$.

11.- Setia M S, Steinmaus C, Ho C S, Rutherford G W. The role of BCG in prevention of leprosy: a meta-analysis. Lancet Infect Dis 2006; 6: 162-70.

12.- Scollard D M, Joyce M P, Gillis T P. Development of leprosy and type 1 leprosy reactions after treatment with infliximab: a report of 2 cases. Clin Infect Dis 2006; 43: 19-22.

13.- Suneetha S, Sigamoni A, Kurian N, Chacko C J. The development of cutaneous lesions during follow-up of patients with primary neuritic leprosy. Int J Dermatol 2005; 44: 224-9.

14.- Beyene D, Aseffa A, Harboe M, Kidane D, Macdonald M, Klatser P R, et al. Nasal carriage of Mycobacterium leprae DNA in healthy individuals in Lega Robi village, Ethiopia. Epidemiol Infect 2003; 131: 8418.

15.- Kaur C, Thami G P, Mohan H. Lucio phenomenon and Lucio leprosy. Clin Exp
Dermatol 2005; 30: 525-7.

16.- Britton W J, Lockwood D N. Leprosy. Lancet 2004; 363: 1209-19.

17.- Cho S N, Cellona R V, Villahermosa L G, Fajardo T T Jr, Balagon M V, Abalos R M, et al. Detection of phenolic glycolipid I of Mycobacterium leprae in sera from leprosy patients before and after start of multidrug therapy. Clin Diagn Lab Immunol 2001; 8 : 138-42.

18.- Martínez A N, Britto C F, Nery J A, Sampaio E P, Jardim M R, Sarno E N, et al. Evaluation of real-time and conventional PCR targeting complex 85 genes for detection of Mycobacterium leprae DNA in skin biopsy samples from patients diagnosed with leprosy. J Clin Microbiol 2006; 44: 3154-9.

19.- Agrawal S, Agarwalla A. Dapsone hypersensitivity syndrome: a clinicoepidemiological review. J Dermatol 2005; 32: 883-9.

20.- Jadhav V H, Patki A H, Mehta J M. Comparison of two multidrug regimens in multibacillary leprosy. Indian J Lepr 1992; 64: 501-4.

21.- Smith C M, Smith C S. Chemoprophylaxis is effective in the prevention of leprosy in endemic countries: a systematic review and meta-analysis. J Infect 2000; 41: 137-42. 